\title{
The Use of Health Information Exchange to Augment Patient Handoff in Long-Term Care: A Systematic
}

\section{Review}

\author{
Clemens Scott Kruse ${ }^{1}$ Gabriella Marquez ${ }^{1} \quad$ Daniel Nelson $^{1} \quad$ Olivia Palomares $^{1}$ \\ ${ }^{1}$ School of Health Administration, Texas State University, San Marcos, \\ Texas, United States \\ Address for correspondence Clemens Scott Kruse, PhD, MHA, MSIT, \\ MBA, FACHE, School of Health Administration, Texas State University, \\ Appl Clin Inform 2018;9:752-771. \\ CHP Room 2050A, 601 University Drive, San Marcos, TX 78666 , \\ United States (e-mail: scottkruse@txstate.edu).
}

\begin{abstract}
Keywords

- health information exchanges

- clinical data

- older patients

- information technology

- communications

Background Legislation aimed at increasing the use of a health information exchange (HIE) in healthcare has excluded long-term care facilities, resulting in a vulnerable patient population that can benefit from the improvement of communication and reduction of waste.

Objective The purpose of this review is to provide a framework for future research by identifying themes in the long-term care information technology sector that could function to enable the adoption and use of HIE mechanisms for patient handoff between long-term care facilities and other levels of care to increase communication between providers, shorten length of stay, reduce 60-day readmissions, and increase patient safety.

Methods The authors conducted a systematic search of literature through CINAHL, PubMed, and Discovery Services for Texas A\&M University Libraries. Search terms used were ("health information exchange" OR "healthcare information exchange" OR "HIE") AND ("long term care" OR "long-term care" OR "nursing home" OR "nursing facility" OR "skilled nursing facility" OR "SNF" OR "residential care" OR "assisted living"). Articles were eligible for selection if they were published between 2010 and 2017, published in English, and published in academic journals. All articles were reviewed by all reviewers and literature not relevant to the research objective was excluded.

Results Researchers selected and reviewed 22 articles for common themes. Results concluded that the largest facilitator and barrier to the adoption of HIE mechanisms is workflow integration/augmentation and the organizational structure/culture, respectively. Other identified facilitator themes were enhanced communication, increased effectiveness of care, and patient safety. The additional barriers were missing/incomplete data, inefficiency, and market conditions.

Conclusion The long-term care industry has been left out of incentives from which the industry could have benefited tremendously. Organizations that are not utilizing health information technology mechanisms, such as electronic health records and HIEs, are at a disadvantage as insurers switch to capitated forms of payment that rely on reduced waste to generate a profit.
\end{abstract}

received

April 22, 2018

accepted after revision

July 29, 2018
DOI https://doi.org/

10.1055/s-0038-1670651.

ISSN 1869-0327. (c) 2018 Georg Thieme Verlag KG

Stuttgart · New York
License terms

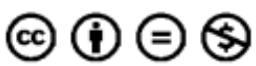




\section{Background and Significance}

Age is positively associated with chronic conditions and utilization of health resources. As 1.3 million of the population in America age, the chances of them contracting one or more chronic conditions increase as do the chances of some of them entering the 16,100 nursing homes in our nation, and some of these conditions are best managed through an electronic information-based record system. ${ }^{1,2}$ As the complexity of care increases beyond what a residential or community-based care facility can manage, a transition of care must occur to a higher level of care. During these transitions, communication must occur at multiple levels to ensure a smooth transition for both patient and receiving organization, smooth transfer of records between organizations, maximization of outcomes, a minimization of length of stay, and, if possible, a reduction of readmissions to care within 30 or 60 days. ${ }^{3,4}$ Inefficiencies during transfer can cause unnecessary rehospitalization as well as more complicated medical outcomes. ${ }^{4}$ Care transitions are often complicated with lack of interoperability of record systems, high utilization at either end, and possibly the patient's reduced ability to participate in the communication portion of the transition of care.

Health information exchange (HIE) is both a noun and a verb: It is both the point of exchange and it also refers to the electronic sharing of health information across organizational boundaries. ${ }^{5}$ The use of electronic HIE has modified how healthcare organizations view patient handoff. ${ }^{1} \mathrm{HIE}$ is a small subset of health information technology (HIT), which has gained large attention over the last decade through various efforts such as in the United States by its Office of the National Coordinator. Long-term care (LTC) organizations such as nursing homes, skilled nursing facilities (SNFs), and assisted living are laggards in the healthcare industry for HIT adoption, and the healthcare industry has been a laggard in IT adoption compared with other industries. ${ }^{5}$ As such, there is a gap in the literature about this important topic. Because the U.S. healthcare industry has been a laggard in technological adoption, legislation aimed at increasing the rate at which organizations make use of electronic health records (EHRs) systems was enacted in 2009. The Health Information Technology for Economic and Clinical Health Act of 2009 (HITECH) allocated more than $\$ 560$ million for states to develop and refine HIE capabilities within their boundaries. ${ }^{6}$ None of the incentives outlined in legislation aimed at increasing interoperability were directed toward the approximately 16,100 nursing homes nationwide, and although these organizations face substantial barriers to even initial EHR adoption, states could have included LTC organizations in their HIE efforts, but very few did. ${ }^{5}$

Previous research briefly examined HIE. A recent crosssectional analysis of secondary data of all U.S. acute-care hospitals reported that of the 1,991 hospitals reporting readmission data, $57.2 \%(1,139)$ of these facilities make some level of effort to exchange clinical data with LTC facilities. ${ }^{3}$ Those that reported some exchange of data with LTC facilities were more than likely to report qualification for meaningful use (odds ratio [OR], $1.87 ; p=0.01$ for stage 1 and OR, 2.05; $p<0.01$ for stage 2 ). While this study analyzed 1,991 organizations, it is unclear how many LTC facilities were involved at the other end of the exchange.

HIE can be used for fiscal reasons. Adopting HIT is part of a complicated attempt to manage the present unsustainable cost growth in healthcare. ${ }^{7}$ In addition to the cost function, HIT is necessary for HIE which can play a part in patient handoff between levels of care. A lack of clear communication at patient handoff results in increased morbidity, costs, and hospital readmissions. ${ }^{8}$ A barrier to HIE adoption is lack of interoperability, lack of funding, and lack of willingness on the part of organizations to change. ${ }^{1}$ It is evident that there are multiple factors pushing for an increase in the interoperability of EHR systems: the adoption of HIT and the diffusion of HIE.

HIE can be used for safety reasons. The increase of nosocomial infections and readmissions at patient handoff has plagued organizations as ill, and even immunocompromised individuals are transferred between organizations. Health information exchanges have emerged as an information-based approach that increases the amount of information transferred at patient handoff. ${ }^{9}$ Medicare no longer reimburses organizations for unplanned readmissions within 30 days for patients with acute myocardial infarction, congestive heart failure, or pneumonia. ${ }^{10}$ This creates an urgent need for organizations to manage information efficiently to reduce the risk of patient readmissions at patient handoff. ${ }^{9}$ Regardless, post-acute care providers as well as LTC organizations have the responsibility to ensure that the transfer of a patient is as risk free as possible.

A gap in the literature exists. The increased use of EHR systems serves as an impetus in the use of HIE. However, little is known about hospital-to-LTC utilization of HIE at a nationwide level. ${ }^{8}$ Several organizations have taken initiative to increase the interorganizational transfer of information to produce successful patient handoffs. Organizations such as the Continuum of Care Improvement Through Information New York have set out to engage organizations and relay pertinent data to relevant stakeholders. ${ }^{9}$ The state of New York has made the largest state-based investment for the improvement of EHR and HIE systems. These improvements serve as a part of the 2005 Healthcare Efficiency and Affordability Law for New Yorkers. The program has invested 440 million dollars in HIT mechanisms to reduce the cost of care for patients. ${ }^{11}$ There is currently not a comprehensive record of all mechanisms nationwide which creates a gap in the literature. Further, some extensive research needs to be conducted across the nation to thoroughly explore the topic and publish best practices by those doing it well, particularly for the rest who have not implemented HIE.

The systematic literature review is the appropriate mechanism to fill this gap in the literature because it has been described as "the most reliable source of evidence to guide clinical practice," it provides a complete overview and analysis of primary research directed toward one research end, and it is often required as the basis of funded research. ${ }^{12}$ Systematic reviews often "include a broad range of relevant studies that have been undertaken and provide a detailed critical appraisal and synthesis of the individual studies."13 
They are "used increasingly to inform medical decision making, plan future research agendas, and establish clinical policy [;] systematic reviews may strengthen the link between best research evidence and optimal health care."14 A systematic literature review is appropriate to set a foundation for an extensive study to establish best practices in preparation for the doubling of the population of adults aged 65 years and older will rapidly increase health care costs because they are more likely to use healthcare services including long-term care.

\section{Objective}

The purpose of this systematic review is to identify the HIE mechanisms currently in place in LTC institutions as well as recognize barriers to the adoption of a HIE that increases transparency between organizations that transfer patients during care for admission into higher level of care facilities. The LTC organizations under study are nursing homes, SNFs, residential care, and assisted living. The rationale behind this systematic review is to provide a framework for future research by identifying the themes prevalent in the literature. In addition, this research will attempt to define key terms and themes for use in future research.

\section{Methods}

Protocol, Eligibility Criteria, and Information Sources Specific reporting and execution protocols were chosen for this review. The review followed the Preferred Reporting Items for Systematic Reviews and Meta-Analyses (PRISMA) proto$\mathrm{Col}^{15}$ (see - Fig. 1) and it was conducted using techniques from the Assessment for Multiple Systematic Reviews (AMSTAR) standard. ${ }^{16}$ Literature gathered for this review was obtained from three separate databases: Cumulative Index of Nursing and Allied Health Administration Literature (CINAHL) Complete, PubMed (which queries MEDLINE), and Discovery Services for Texas A\&M University Libraries. The reviewers combined key terms from the U.S. Library of Medicine's Medical Subject Headings (MeSH) with Boolean operators in all three databases to identify articles. Criteria focused on inpatient residential care organizations and the use (or nonuse) of a HIE. Analyzed literature covers a broad array of geographic regions as well as organizations at various levels of meaningful use standards. Search criteria emphasized the transition of care from acute care to a LTC organization.

\section{Search and Study Selection}

The search terms and applicable Boolean terms were as follows: ("health information exchange" OR "healthcare information exchange" OR "HIE") AND ("long term care" OR "long-term care" OR "nursing home" OR "nursing facility" OR "skilled nursing facility" OR "SNF" OR "residential care" OR "assisted living"). These terms were deliberately chosen by examining the index list in the MeSH. Search parameters included English articles published from January 2010 to December 2017. This timeframe was selected because it was post-HITECH Act. While there were many HIE efforts prior to HITECH, the EHR adoption rates significantly increased afterward due to the meaningful-

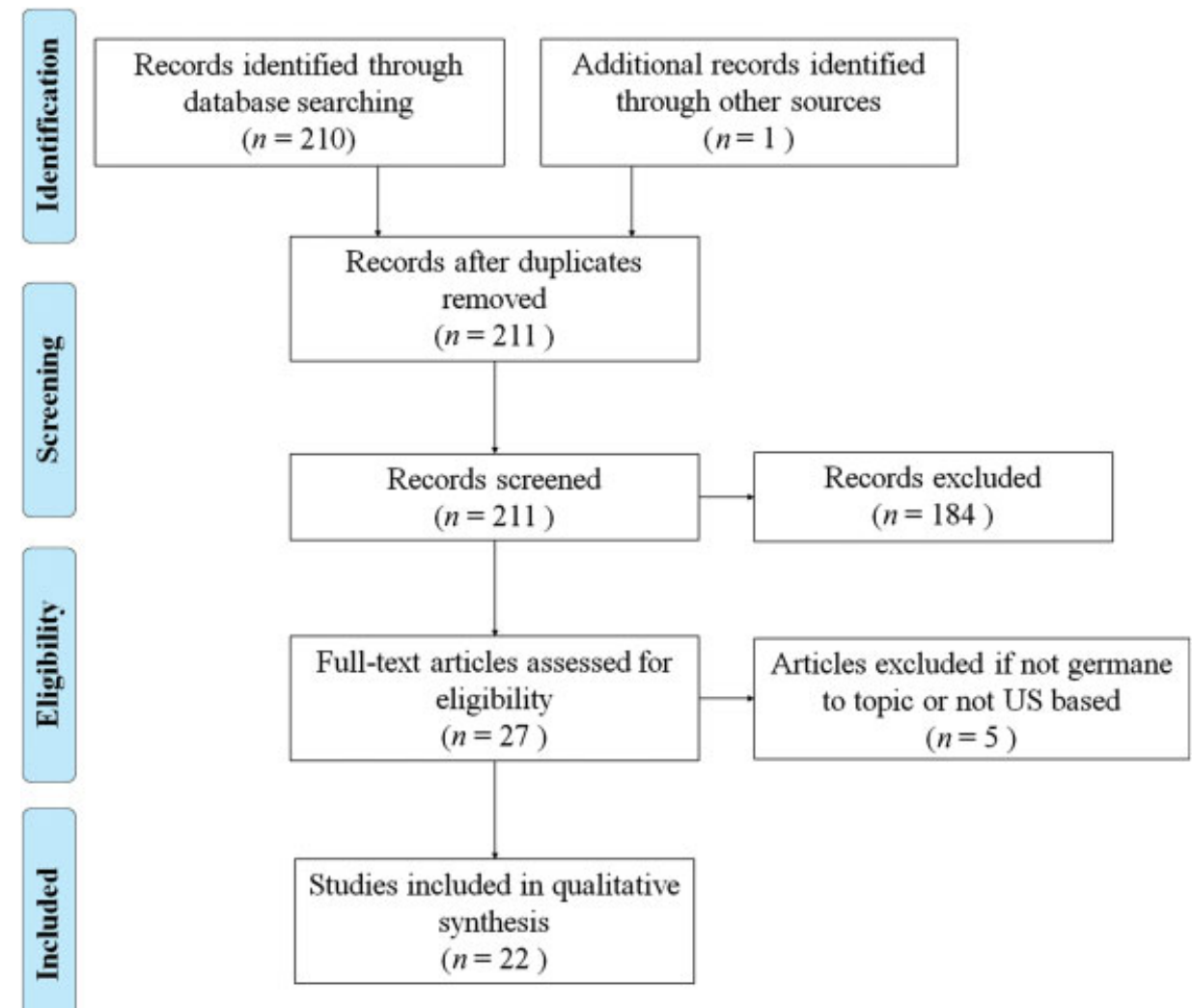

Fig. 1 PRISMA checklist. 
use incentives. Organizations with EHRs exchange clinical data through HIE two and a half times more than their non-EHR counterparts. ${ }^{5}$ Therefore, it was imperative to observe HIE activity during this catalyst stage of EHR adoption. - Fig. 2 demonstrates the literature review search process that shows the inclusive and exclusion criteria.

The initial search resulted in 210 articles. We filtered the search for the last 7 years as well as full-text, English-only, and academic journals (to maintain quality of results). This removed all but 39 results. These 39 results were entered on piloted forms for consistency of review. Using a technique from AMSTAR, we assigned abstracts of these 39 articles to all group members in way that ensured each abstract was assessed for its germane nature by at least two reviewers. This process removed an additional 14 articles. Our final group for analysis was 22 articles.

\section{Data Collection Process and Data Items}

The authors reviewed each article in the same manner as the abstracts and determined the barriers and facilitators for the use of HIE to augment patient handoff in LTC. Piloted forms extracted similar data from each article: authors, year of publication, journal, country of publication, sample size, study design, signs of bias, limitations of study, facilitators for adoption, barriers to adoption, and a column for general comments. Facilitators were defined as characteristics or environmental factors that enabled the adoption and use of HIE between LTC and other levels of care. Barriers were defined as characteristics or environmental factors that serves as an obstacle or impediment to the adoption or use of HIE between LTC and other levels of care. The reviewers also examined potential bias and limitations of each article. Reviewers once again held a consensus meeting to discuss and compile observations as well as resolve any differences in observation.

\section{Risk of Bias in Individual Studies and Across Studies}

Reviewers recorded observed bias and potential limitations for each article analyzed as well as across all articles. These observations were recorded on the literature matrix along with the rest of the analysis. All results were recorded on a common table and compiled for discussion. - Appendix A details bias and limitations of studies.

\section{Synthesis of Results and Additional Analysis}

The final consensus meeting consisted of overall discussion of observations and inferences. Once all observations were compiled among all reviewers, narrative analysis and sense making were conducted to combine similar terms; ${ }^{17}$ for example, one set of observations was "workflow integration" and another was "workflow augmentation." Because both had to do with an action on workflows, we combined these into one theme. "Organizational structure" and "organizational culture" were also similar because they were both attributes of organizations. "Missing data" and "incomplete data" both described deficiencies of data, so they were combined. Finally, "privacy" and "security" were combined because these are often combined when discussing patient information. Based on the themes in the narrative analysis, affinity tables were created to synthesize themes for discussion.

\section{Results}

\section{Study Selection}

Our literature search narrowed 210 results down to 22 by assessing each for suitability to our research objective. ${ }^{2-9,18-30}$
CINAHL complete

("health information exchange" OR "healthcare
information exchange" OR "HIE") AND ("long term
care" OR "long-term care" OR "nursing home" OR
"nursing facility" OR "skilled nursing facility" OR
"SNF" OR "residential care" OR "assisted living") $(n=13)$

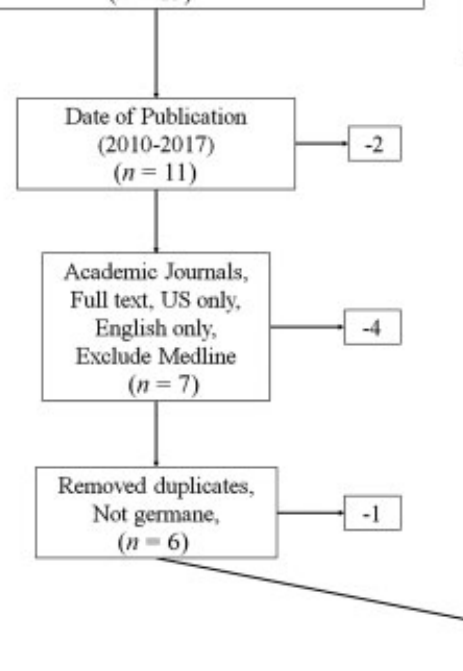

PubMed information exchange" OR "HIE") AND ("long term care" OR "long-term care" OR "nursing home" $O R$ "nursing facility" OR "skilled nursing facility" OR "SNF" OR "residential care" OR "assisted living") $(n=27)$

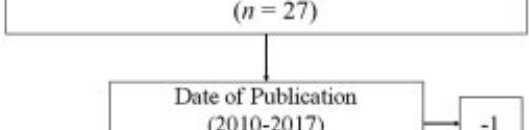
(2010-2017) $(n=26)$
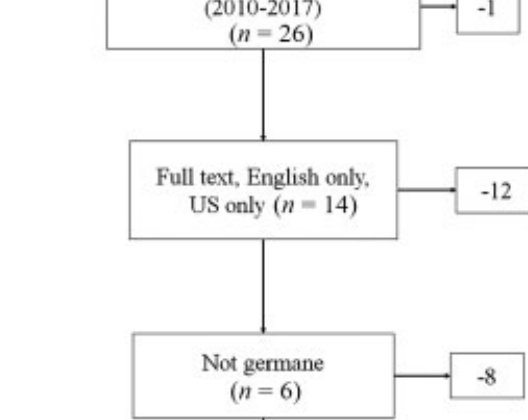

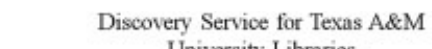
University Libraries

("health information exchange" OR "healthcare information exchange" OR "HIE") AND ("long term care" $O R$ "long-term care" $O R$ "nursing home" OR "nursing facility" OR "skilled nursing facility" OR "SNF" OR "residential care" OR "assisted living") $(n=170)$
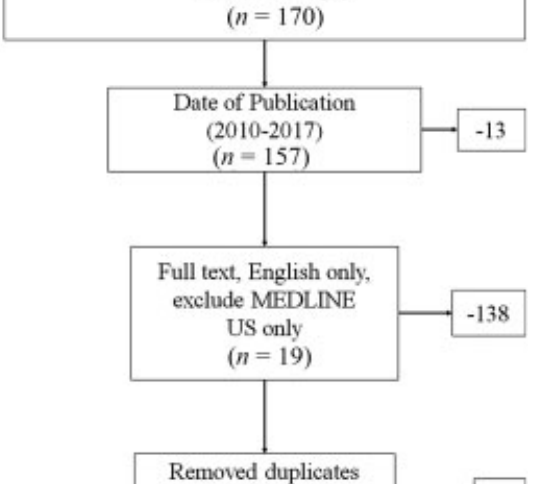
$(n=10)$

Fig. 2 Search criteria with inclusive and exclusion criteria. 
We excluded 16 results because they were outside our target date range of 2010 to 2017. Another 157 results were excluded when we used the filters of English-only, full-text, and academic journals. The abstracts of the remaining 40 articles were assessed for suitability by multiple reviewers which reduced the final group to 22. A kappa statistic was calculated to assess the similarity in selection by reviewers. ${ }^{31}$ It calculated to 0.99 , which is near-perfect agreement. ${ }^{32}$ See - Fig. 2 for the selection process. See - Appendix A for detailed calculations of the kappa statistic.

\section{Study Characteristics}

From each study, reviewers extracted observations of facilitators, barriers, potential bias, and limitations. These are summarized in - Table 1. We sorted the group of articles by date, most recent to oldest. There were three from 2017, 2,3,19 three from 2016, ${ }^{4,20,21}$ six from 2015, ${ }^{5,18,22-24}$ five from $2014,,^{5,9,26-28}$ three from $2013,,^{7,8,29}$ one from $2012,{ }^{30}$ and one from $2011 .^{11}$

\section{Risk of Bias within Studies}

The biases observed within studies were selection bias, 3,27 workplace bias, ${ }^{2}$ self-report bias, ${ }^{4,26,30}$ data collection bias, ${ }^{5,9}$ cognitive bias, ${ }^{8}$ and nonresponse bias. ${ }^{8}$ The bias observed did not appear strong enough to discount any of the articles in the group for analysis.

\section{Results of Individual Studies}

Articles analyzed identified a variety of facilitators and barriers, but common themes could be traced through the group. A detailed list of all observations and how they line up with themes can be seen in -Appendix B. Facilitators are illustrated in -Table 2 . The asterisk $\left({ }^{*}\right)$ by the reference number indicates that the theme occurred within the article multiple times.

About $33 \%$ of the facilitators were captured with six themes. The themes organizational structure/culture ${ }^{3,4,18,21,25,28,29}$ was observed seven times. These articles expressed issues such as the organization characteristics such as bed size, location, ownership, office or hospital based, system affiliation, or a flexible organization culture that enabled HIE. Accountable care organization incentive structures also enable HIE with LTC. The theme workflow integration/augmentation ${ }^{6,19 * 21,27,28}$ was observed six times out of 39 occurrences. These articles described various aspects of workflow integration and or augmentation. External data are effective only if it can be

Table 1 Facilitators and barriers

\begin{tabular}{|l|l|}
\hline Facilitators & Barriers \\
\hline Ease of data transfer & Inefficiency \\
\hline Reduce healthcare costs & Cost \\
\hline Government funding & Low usage/Adoption \\
\hline $\begin{array}{l}\text { Adoption of EHR is likely to } \\
\text { use HIE }\end{array}$ & Competing organizations \\
\hline
\end{tabular}

Abbreviations: EHR, electronic health record; HIE, health information exchange.
Table 2 Affinity matrix of facilitator themes in the literature

\begin{tabular}{|c|c|c|}
\hline \multicolumn{3}{|l|}{ Facilitators } \\
\hline Theme & References & $\begin{array}{l}\text { No. of } \\
\text { occurrences }\end{array}$ \\
\hline $\begin{array}{l}\text { Organizational } \\
\text { structure/culture }\end{array}$ & $3,4,18,21,25,28,29$ & 7 \\
\hline $\begin{array}{l}\text { Workflow integra- } \\
\text { tion/augmentation }\end{array}$ & $6,19^{*}, 21,27,28$ & 6 \\
\hline $\begin{array}{l}\text { Increase effective- } \\
\text { ness of care }\end{array}$ & $2,19,22,24,27$ & 5 \\
\hline $\begin{array}{l}\text { Enhance } \\
\text { communication }\end{array}$ & $5,9,23,27$ & 4 \\
\hline Adoption of EHR & $4,5,20,28$ & 4 \\
\hline Proper funding & $4,7,11$ & 3 \\
\hline Patient safety & 25,27 & 2 \\
\hline Ease of data transfer & 2,9 & 2 \\
\hline Efficiency & 11,16 & 2 \\
\hline Market conditions & 13,31 & 2 \\
\hline \multirow[t]{2}{*}{$\begin{array}{l}\text { Reduce healthcare } \\
\text { cost }\end{array}$} & 10,19 & 2 \\
\hline & & 39 \\
\hline
\end{tabular}

Abbreviation: EHR, electronic health record.

${ }^{*}$ The asterisk by the reference number indicates that the theme occurred within the article multiple times.

integrated into the clinical workflow. ${ }^{33}$ When an organization designs a workflow, it must take a human-systems approach, which is to say that the human is the embedded component of a system that supports people through a recognition of human capabilities, limitations, and performance needs. When the system is designed this way, outcomes are improved for patient, provider, and organization. ${ }^{34}$ Some observed that adoption of HIE for patient transfer integrated with their existing workflows, and they observed that an organization with a supportive culture was conducive to a successful implementation. The asterisk $\left(^{*}\right)$ next to the reference number indicates that more than one facilitator was observed for that theme in the same article. For instance, one article pointed out that HIE integrated nicely with their existing workflows, and it improved or augmented their workflows as well. This was seen in both their billing cycle and their documentation. ${ }^{19}$ Faster billing was observed multiple times and was captured under the same theme. ${ }^{6}$ Articles described policy initiatives that incentivized better coordination of transfer and promoted information sharing. ${ }^{6,21}$ A receptive and supportive leadership that supports IT acceptance is influenced by performance expectancy, effort expectancy, social influence, and voluntariness. $^{12,18}$ LTC facilities pointed out that HIE was necessary to create continuity-of-care documentation that was greatly appreciated by the receiving organizations. ${ }^{25}$ It was also noted that nonprofit organizations were more likely to adopt HIE than their for-profit counterparts. ${ }^{29}$

HIE is attributed to improving the effectiveness of care. $2,19,22,24,27$ Five articles discussed this attribute. The 
use of HIE was shown to decrease readmissions and decrease adverse events. ${ }^{19,22,27}$ Others intimated that HIE allowed more in-depth, long-term views into a patient's history, particularly those with complex care. ${ }^{2,24}$ These five occurrences represented $13 \%$ of the total occurrences observed.

Four articles mentioned that HIE improved their communication both within and external to their own organization. ${ }^{5,9,23,27}$ HIE was named responsible for distributing patient status to the entire clinical team, and because the coordination of care involves many people, HIE has been adopted to ease the human resources burden to this task. ${ }^{5,23}$ Enhanced communication helps fill a gap where the lack of communication is a leading cause of error in healthcare. ${ }^{27}$ The United States is not the only country to encourage HIE. In the United Kingdom, HIE is used to enhance communication as well. ${ }^{35}$ These four occurrences represented $10 \%$ of all occurrences.

Adoption of the $E H R^{2,9}$ was also observed four times, representing another $10 \%$ of the total observations. This observation noted that the adoption of HIE has been easier along with the adoption of the EHR. HIE was attributed for safer transitions of care, which is where errors most occur. Of private practices that responded to one survey, $15 \%$ of them were actively sharing information with LTC. ${ }^{27}$ Proper funding was mentioned three times in the literature ${ }^{4,7,11}$ representing $8 \%$ of all occurrences.

The last five themes were each mentioned twice in the literature, accounting for $25 \%$ of all occurrences. Patient safety ${ }^{25,27}$ was mentioned because organizations liked the reduction of duplicate testing, because a reduction in duplication increased communication, which may lead to fewer medical errors, and improved patient outcomes. The others were ease of data transfer, ${ }^{2,9}$ efficiency, ${ }^{11,16}$ market conditions, ${ }^{13,31}$ and reduce healthcare cost..$^{10,19}$ The proper funding theme spoke of the cost of both EHR and HIE capabilities; some of these organizations were included in state efforts of HIE, and therefore they were able to afford the capability. Articles that highlighted the theme of ease of data transfer spoke of their perception that exchanging clinical data through HIE is easier than through fax or courier. Issues of efficiency were similar to issues of workflow, but they did not expressly use this term. We could have combined these into the workflow theme. The theme of market conditions spoke of concerns that resources spent on HIE were necessary because other organizations in their market were using $\mathrm{HIE}$, and therefore to compete, they felt obligated to invest in the capability. Finally, the theme of reduce healthcare cost referred to the ongoing efforts to reduce the cost of care. Many organizations feel that a continued investment in IT will only increase the cost of care rather than realizing the cost saving and patient safety effects of HIE by preventing duplicate test, improving communication, saving time, and improving outcomes. The articles highlighted in this group realized the latter.

Five themes represented approximately $81 \%$ of all occurrences (39/48) of barriers. These are illustrated in - Table 3. The asterisk $\left({ }^{*}\right)$ by the reference number indicates that the theme occurred within the article multiple times.
Table 3 Affinity matrix of barrier themes in the literature

\begin{tabular}{|l|l|l|}
\hline Barriers & References & Occurrences \\
\hline Theme & $5^{*}, 4,6,7,11,19,25,26$ & 10 \\
\hline Cost & $2,4,5,9,18,21,22,26^{*}$ & 9 \\
\hline $\begin{array}{l}\text { Organizational } \\
\text { structure/ } \\
\text { culture }\end{array}$ & $2,3,23,24,27,30$ & 6 \\
\hline $\begin{array}{l}\text { Missing/incom- } \\
\text { plete data }\end{array}$ & $26^{*}, 28$ & 6 \\
\hline Inefficiency & $5,24,26$ & 4 \\
\hline $\begin{array}{l}\text { Market } \\
\text { conditions }\end{array}$ & 26,29 & 2 \\
\hline $\begin{array}{l}\text { Lack of data } \\
\text { standards }\end{array}$ & 26,29 & 2 \\
\hline $\begin{array}{l}\text { Privacy and/or } \\
\text { security }\end{array}$ & 64,26 & 2 \\
\hline Lack of training & 6,8 & 24 \\
\hline $\begin{array}{l}\text { Legal } \\
\text { environment }\end{array}$ & & 2 \\
\hline
\end{tabular}

${ }^{*}$ The asterisk by the reference number indicates that the theme occurred within the article multiple times.

Cost was identified as the second most often mentioned: 10 out of 44 occurrences or $23 \% .^{5 *, 4,6,7,11,19,25,26}$ These articles pointed out the lack of incentives such as the meaningful use program ${ }^{5,19,25}$ or state and local funding. ${ }^{7,11}$ Others pointed out that LTC organizations often do not have the budget for either the acquisition or upkeep costs that are required for such an IT implementation., ${ }^{4,5}$

Organizational structure was observed in 9 of the 44 occurrences $(20 \%)$. $2,4,5,9,18,21,22,26 *$ We did combine organizational culture issues along with organizational structure. The organizations that were the subjects of these articles were not conducive to change or they did not embrace the new technology of HIE, which are cultural issues. Some articles highlighted that their organizations were not suited for even an EHR and the high turnover in LTC organizations is not conducive to an IT implementation like the adoption of HIE. ${ }^{21}$ One article stated that the largest barrier to the adoption of HIE was a necessary cultural change. ${ }^{22}$ Another mentioned that it did not have sufficient stakeholder buy-in. ${ }^{9}$

The next barrier mentioned most often was the missing, incomplete, or inaccurate data associated with HIE. 2,3,23,24,27,30 This was found in 6 of the 44 occurrences, or $14 \%$. These articles pointed out the importance of the correct billing codes for prompt reimbursement, and if the data are not accurate or complete in the transfer, then a delay or penalty will follow, and that comorbidities can complicate the codes. ${ }^{23}$ One article mentioned that unique EHR systems that are not interoperable create data silos because they cannot share information. ${ }^{24}$

Six articles pointed out a perception of inefficiencies that HIE would cause their organization. ${ }^{2,4,8,9,19,26}$ These articles discussed a misalignment of workflows, ${ }^{9,26}$ a communication 
barrier, ${ }^{5,8,19}$ and overburdened staff which would serve as barriers to their adoption of HIE. ${ }^{31}$ These represented $14 \%$ of all occurrences of barriers listed in the literature.

The barrier of market conditions was identified four times in the literature, representing $9 \%$ of all occurrences. ${ }^{26 *}{ }^{28}$ These articles expressed concern about the technological maturity on HIE, and the level of both vendor and health plan participation in HIE. They felt that there was insufficient saturation of HIE in their market to compel them toward the technology.

The other four themes appeared in the literature $20 \%$ of what was analyzed. These themes were lack of data standards, ${ }^{5,24,26}$ which refers to over 300 vendors of EHRs and no one common national solution to sharing data; privacy/ security concerns, ${ }^{26,29}$ which highlighted the constant fear that some organizations can operate with in regard to the security of clinical data and potential fines from both state and federal government; lack of training, ${ }^{6,8}$ which refers to constant change of technology and how the training for these changes is often the responsibility of the organization; and legal environment, ${ }^{26,29}$ which parallels the issue of privacy/ security concerns because often part of the remedy for data breach is credit monitoring and other monetary damages and time in court.

\section{Additional Analysis}

-Tables 2 and 3 illustrate the identified themes throughout the literature for both facilitators and barriers to the adoption of HIE to support patient handoff between levels of care. We also evaluated these themes in terms of internal versus external factors, as depicted in -Tables 4 and 5 . These themes are listed in the same order as previously listed: most often mentioned to least often mentioned. As depicted, the facilitators list only 2 of 11 themes (18\%) as external to

Table 4 Facilitator themes with internal/external association

\begin{tabular}{|l|l|}
\hline Facilitators & \\
\hline Theme & Internal \\
\hline $\begin{array}{l}\text { Workflow integration/ } \\
\text { augmentation }\end{array}$ & Internal \\
\hline $\begin{array}{l}\text { Organizational structure/ } \\
\text { culture }\end{array}$ & Internal \\
\hline Enhance communication & Internal \\
\hline $\begin{array}{l}\text { Increase effectiveness of } \\
\text { care }\end{array}$ & Internal \\
\hline Patient safety & Internal \\
\hline Adoption of EHR & External \\
\hline Proper funding & Internal \\
\hline Ease of data transfer & Internal \\
\hline Efficiency & External \\
\hline Market conditions & Internal \\
\hline Reduce healthcare cost
\end{tabular}

Abbreviation: EHR, electronic health record.
Table 5 Barrier themes with internal/external association

\begin{tabular}{|l|l|}
\hline Barriers \\
\hline Theme & \\
\hline Organizational structure/culture & Internal \\
\hline Cost & External \\
\hline Missing/incomplete data & Internal \\
\hline Inefficiency & Internal \\
\hline Market conditions & External \\
\hline Lack of data standards & External \\
\hline Privacy and/or security & External \\
\hline Lack of training & Internal \\
\hline Legal environment & External \\
\hline
\end{tabular}

the organization. That is to say, only two items listed are beyond the control of the organization: proper funding, mentioned $7 \%$ of all occurrences, and market conditions, mentioned $5 \%$ of all occurrences. The barriers, however, show five of nine themes (55\%) as external. These themes were cost, market conditions, lack of data standards, privacy/ security, and the legal environment, mentioned $21,8,6,4$, and $2 \%$ of all occurrences, respectively.

A brief analysis of the quality of methodology and overall study design can be found in -Appendix C. This appendix was created as part of the piloted forms, but it did not add significant content for the review.

\section{Discussion}

\section{Summary of Evidence}

Our literature search identified 26 articles that were pertinent to our research objective. From these 26 articles, we identified facilitators and barriers identified in the literature. Several of the facilitators and barriers were similar; so, we created themes to capture the essence of the details identified by the literature. We identified 11 themes for facilitators and 9 themes for barriers each listed 39 and 44 times, respectively.

Healthcare leaders should recognize the top facilitators and leverage them for future implementation. These leaders have control over a strong majority of the themes identified. Create an organizational culture that is conducive to the adoption of HIE recognizing that others have easily integrated it into their existing workflows. Leaders often look for ways to increase communication both within and external to the organization, and several articles identified HIE as a way to augment both. Leaders should also recognize that an HIE implementation will take time and should emphasize both process improvement and training of users before undertaking it.

Policy makers should examine the barriers identified in this review. LTC should be included in future financialincentive programs to help offset the cost of implementation. Policy makers should also help set market conditions that are 
most conducive to HIE adoption and continue to develop clear data standards that protect privacy and promote security for the industry.

The reviewed articles indicated a primarily optimistic approach to the future implementation of HIEs in patient handoff. A pilot study implementing a form of HIE between five Oklahoma LTC facilities and their corresponding emergency departments indicated a decrease in the number of readmissions throughout the duration of the 20-month study. $^{22}$ This decrease correlates with the shift in the U.S. healthcare system to a patient-centered, value-based approach that emphasizes HIT as a tool to improve care. ${ }^{22}$

The overall health of the patient population admitted into LTC facilities must be considered as well. Elderly patients are more likely than the rest of the population to take multiple prescriptions, have comorbidities, and see a multitude of providers. ${ }^{22}$ With the multiple opportunities for error that exist because of the health of the geriatric population, organizations and patients alike can benefit from the accurate transfer of information.

Nearly one in four individuals in the United States suffers from multiple conditions, while one-half of the population suffers from a chronic condition. The percentage of the population who suffers from at least one chronic condition increases to $75 \%$ among older adults. ${ }^{5}$ The successful management of these conditions is contingent on the collaboration of multiple stakeholders throughout the course of patient care. ${ }^{23}$ Thus, comprehensive and accurate information is required. A study of 20 clinical staff from a U.S. Midwestern long-term post-acute care facility (LTPAC) found that providing LTPAC staff with a more in-depth view of a patient's condition aids the course of care beyond the acutecare setting. Medication errors such as the erroneous continuation of an antibiotic can be prevented if proper information is available. ${ }^{5}$ In many countries, the LTPAC covers services such as LTC hospitals, nursing homes, SNFs, and residential care. ${ }^{36}$

The end goal of the accurate and efficient transfer of information through an HIE must be articulated as a need for LTC facilities. The concept for an HIE comes in many forms and requires the cooperation of organizational stakeholders. Health information exchanges can aid in the treatment and billing of chronic care coordination. The proper course of action for chronic care coordination requires open communication between pertinent providers to ensure that conditions are properly treated. ${ }^{23}$ For now, many LTC facilities are focused on the proper implementation of EHRs, a concept that the rest of the healthcare world has long since implemented due to the HITECH Act of 2009. LTC facilities that have implemented EHRs utilize HIE mechanisms at a rate of 2.5 times more than their non-EHR counterparts. ${ }^{5}$ Among a cross-sectional study across all New York State nursing homes, most information was exchanged with pharmacies and laboratories. The state of New York has invested a considerable amount of time on both research and implementation of HIE mechanisms in nursing homes and the results are encouraging and illustrate the need for initiatives to drive other organizations to invest in HIEs. ${ }^{5}$
For LTC facilities, there are barriers to the implementation of HIEs aside from the complete lack of financial incentives. The integration of software among organizations is a prominent issue, as well as differing information needs. ${ }^{5}$ Compensation for the coordination of chronic care among the geriatric population may not suffice if the cost of generating billing and documentation exceeds the capitated reimbursement for the treatment of the condition. In this regard, HIEs can reduce unnecessary documentation and tests by improving communication among providers and reducing waste. ${ }^{23}$ Healthcare insurers, including the federal and state governments who pay for a majority of healthcare for older adults, must reduce waste to make a profit.

If organizations do not receive the proper and necessary information, the HIE is only an expense and not a benefit. It is imperative that LTC facilities, which can most benefit from the smooth transfer of information, implement HIE mechanisms to stop the unnecessary harm caused by the poor transfer of information.

\section{Limitations}

Limitations stemmed from the nature of current studies, which assess LTC implementation of EHRs and HIEs in general. Because the implementation of HIT in LTC organizations is low compared with other organizations, studies that assess the true notion of patient handoff from acute care to LTC facilities or from LTC facilities to higher levels of care are limited and premature in their scope.

While selection bias is a large threat to any study, we controlled for that threat by adopting techniques from AMSTAR. We ensured multiple reviewers independently evaluated articles and held regular consensus meetings to keep everyone calibrated on the purpose and scope of this review.

One of the filters used in the search for articles was "free text" which eliminated seven articles from consideration. This is a limitation because the impact of those seven articles on the outcome of our systematic literature review is unknown. Additional funding would enable access to these articles and if findings are significantly different, a study should be republished.

Another limitation is that this systematic literature review fell short of a meta-analysis. A sufficient level of original data from the studies was not collected. The science behind the studies analyzed was not sufficiently robust. Our time was short for this round of research, so we held short at the systematic review.

\section{Conclusion}

Health information exchanges are imperative to the future of care in the LTC sector. With the growth of the aging population, organizations must be ready to provide accurate quality care to ensure the well-being of patients. The benefits of an HIE cannot be underestimated, given that the elderly population presents more comorbidities and chronic condition than the rest of the population. Current barriers to the proper implementation of an HIE include network pitfalls and 
competing stakeholder needs, as well as the financial component of implementing a useable HIE.

The status of EHR implementation in LTC facilities varies throughout the country with HIEs lagging even further behind. ${ }^{37}$ Significant research has been conducted on hospital readmissions from the LTC setting, but not enough research has been conducted on successful mechanisms to prevent readmissions. ${ }^{38}$ Future research should focus on the need to increase financial incentives for LTC facilities to implement HIE mechanisms, as well as the actuarial effect of readmissions on LTC facilities as financial penalties become more stringent. This systematic review serves as a good foundation for extensive research to establish best practices.

\section{Multiple Choice Questions}

1. The HITECH Act applied incentives for the adoption of HIT for which of the following organizations?

a. Acute-care hospitals.

b. All size of physician practices.

c. Long-term care.

d. a and b only.

Correct Answer: The correct answer is option d.

2. Which is the largest barrier to the adoption of HIT in LTC organizations?
a. Cost.
b. Privacy/security.
c. Organizational culture.
d. Market conditions.

Correct Answer: The correct answer is option a.

\section{Protection of Human and Animal Subjects}

This study was performed in compliance with the World Medical Association Declaration of Helsinki on Ethical Principles for Medical Research Involving Human Subjects, and was not reviewed by Institutional Review Board because it is exempted, IAW 45CFR46.

\section{Conflict of Interest}

None.

Funding

None.

\section{References}

1 Vest JR, Gamm LD. Health information exchange: persistent challenges and new strategies. J Am Med Inform Assoc 2010;17 (03):288-294

2 Meehan R. Transitions from acute care to long-term care: evaluation of the continued access model. J Appl Gerontol 2017;00 (0): $1-20$

3 Cross DA, Adler-Milstein J. Investing in post-acute care transitions: electronic information exchange between hospitals and long-term care facilities. J Am Med Dir Assoc 2017;18(01):30-34

4 Towne SD Jr, Lee S, Li Y, Smith ML. Assessment of eHealth capabilities and utilization in residential care settings. Health Informatics J 2016;22(04):1063-1075
5 Abramson EL, McGinnis S, Moore J, Kaushal R; HITEC Investigators. A statewide assessment of electronic health record adoption and health information exchange among nursing homes. Health Serv Res 2014;49(1, Pt 2):361-372

6 Filipova AA. Health information exchange capabilities in skilled nursing facilities. Comput Inform Nurs 2015;33(08):346-358

7 MacTaggart P, Thorpe JH. Long-term care and health information technology: opportunities and responsibilities for long-term and post-acute care providers. Perspect Health Inf Manag 2013;10 (Fall):1e

8 Kessler C, Williams MC, Moustoukas JN, Pappas C. Transitions of care for the geriatric patient in the emergency department. Clin Geriatr Med 2013;29(01):49-69

9 Richardson JE, Malhotra S, Kaushal R; HITEC Investigators. A case report in health information exchange for inter-organizational patient transfers. Appl Clin Inform 2014;5(03):642-650

10 Stone J, Hoffman GJ. Medicare hospital readmissions: issues, policy options and PPACA. Congressional Research Service; 2010:1-37

11 Kern LM, Wilcox AB, Shapiro J, et al. Community-based health information technology alliances: potential predictors of early sustainability. Am J Manag Care 2011;17(04):290-295

12 Clarke J. What is a systematic review? Evid Based Nurs 2011;14 (03):64

13 Greaves K. What is a systematic review? Adv Skin Wound Care 2014

14 Cook DJ, Mulrow CD, Haynes RB. Systematic reviews: synthesis of best evidence for clinical decisions. Ann Intern Med 1997;126 (05):376-380

15 Moher D, Liberati A, Tetzlaff J, Altman DG; PRISMA Group. Preferred reporting items for systematic reviews and meta-analyses: the PRISMA statement. Int J Surg 2010;8(05):336-341

16 Shea BJ, Grimshaw JM, Wells GA, et al. Development of AMSTAR: a measurement tool to assess the methodological quality of systematic reviews. BMC Med Res Methodol 2007;7(01):10

17 Reissman CK. Narrative Analysis. Newbury Park, CA: Sage; 1993

18 Alexander GL, Rantz M, Galambos C, et al. Preparing nursing homes for the future of health information exchange. Appl Clin Inform 2015;6(02):248-266

19 Meehan RA, Staley J. 2017. Facilitating long-term care providers' participation in accountable care organizations through health information exchange. Perspectives in Health Information Management, 1-7. Available at:http://search.ebscohost.com/login.aspx? direct=true $\&$ profile $=$ ehost $\&$ scope $=$ site \&authtype $=$ crawler\&jrnl= $15594122 \& A N=124305208 \& \mathrm{~h}=\mathrm{TZesjBNWZQ2ZyhjpFNH}-$ puIJ73B13Pf5XimwZIjiqvAcQi1ygWuyigSP9ubUqzJgqSDYGCXHkuy1aLes7fZB5hw\%3D\%3D\&crl=c. Accessed September 5, 2018

20 Jamoon E, Yang N, Hing E. Adoption of Certified Electronic Health Record Systems and Electronic Information Sharing in Physician Offices: United States, 2013 and 2014. U.S. Department of Health and Human Services. NCHS Data Brief, No. 236; 2016

21 Alexander GL, Popejoy L, Lyons V, et al. Exploring health information exchange implementation using qualitative assessments of nursing home leaders. Perspect Health Inf Manag 2016;13 (Fall):1f

22 Yeaman B, Ko KJ, Alvarez del Castillo R. Care transitions in longterm care and acute care: health information exchange and readmission rates. Online J Issues Nurs 2015;20(03):5

23 Peters SG, Bunkers KS. Chronic care coordination. Chest 2015;148 (04):1115-1119

24 Hill D, Du Fresne LJ, Holder I, Samudio R, Sujana N. Interconnectivity of health information exchanges using patient access number (PAN). ASBBS eJournal 2015;11(01):7-21

25 Hassol A, Goodman L, Younkin J, Honicker M, Chaundy K, Walker JM. Survey of state health information exchanges regarding inclusion of Continuity of Care Documents for long-term postacute care (LTPAC) patient assessment. Perspect Health Inf Manag 2014;11(Fall):1g 
26 Campion TR Jr, Vest JR, Kern LM, Kaushal R; HITEC investigators. Adoption of clinical data exchange in community settings: a comparison of two approaches. AMIA Annu Symp Proc 2014; 2014:359-365

27 Lyngstad M, Hellesø R. Electronic communication experiences of home health care nurses and general practitioners: a cross-sectional study. Studies in Health Technology and Informatics, 201 (Nursing Informatics 2014: East Meets West eSMART+ - Proceedings of the 12th International Congress on Nursing Informatics, NI); 2014:388-394. Doi: 10.3233/978-1-61499-415-2-388

28 Wang T, Wang Y, Moczygemba J. Organizational factors influencing health information technology adoption in long-term-care facilities. Health Care Manag (Frederick) 2014;33(01):30-37

29 Hamann DJ, Bezboruah KC. Utilization of technology by long-term care providers: comparisons between for-profit and nonprofit institutions. J Aging Health 2013;25(04):535-554

30 Wolf L, Harvell J, Jha AK. Hospitals ineligible for federal meaningfuluse incentives have dismally low rates of adoption of electronic health records. Health Aff (Millwood) 2012;31(03):505-513

31 Light RJ. Measures of response agreement for qualitative data: some generalizations and alternatives. Psychol Bull 1971;76(05): 365-377
32 McHugh ML. Interrater reliability: the kappa statistic. Biochem Med (Zagreb) 2012;22(03):276-282

33 Mishuris RG, Yoder J, Wilson D, Mann D. Integrating data from an online diabetes prevention program into an electronic health record and clinical workflow, a design phase usability study. BMC Med Inform Decis Mak 2016;16(01):88

34 Holden RJ, Carayon P, Gurses AP, et al. SEIPS 2.0: a human factors framework for studying and improving the work of healthcare professionals and patients. Ergonomics 2013;56(11): 1669-1686

35 Gaskin S, Georgiou A, Barton D, Westbrook J. Examining the role of information exchange in residential aged care work practices-a survey of residential aged care facilities. BMC Geriatr 2012;12 (01):40

36 U.S. Department of Health and Human Services. Available at: https:// www.healthit.gov/playbook/care-settings/. Accessed April 19, 2018

37 Wei Q Courtney KL. Nursing information flow in long-term care facilities. Appl Clin Inform 2018;9(02):275-284

38 Sockolow PS, Weiner JP, Bowles KH, Abbott P, Lehmann HP. Advice for decision makers based on an electronic health record evaluation at a program for all-inclusive care for elders site. Appl Clin Inform 2011;2(01):18-38

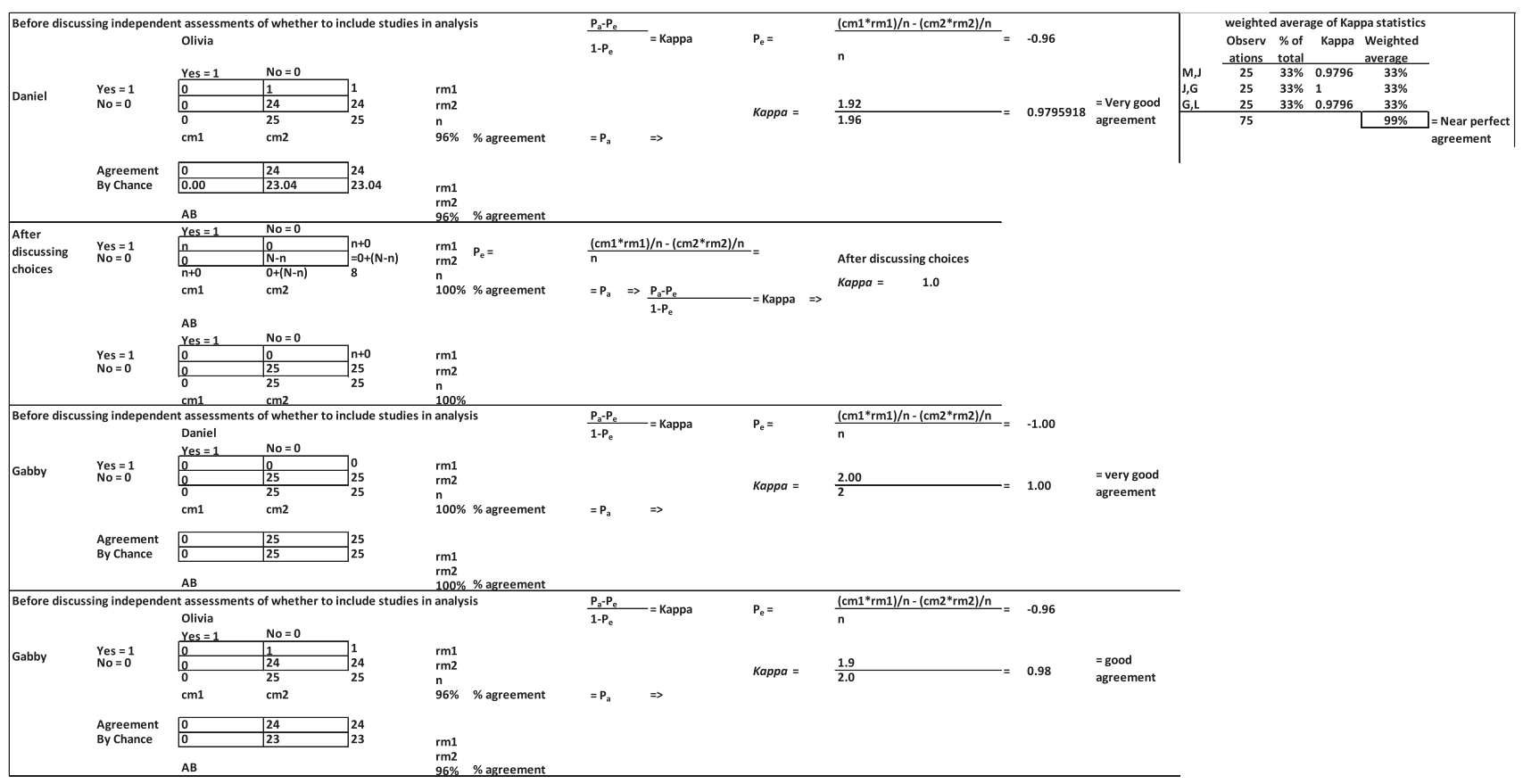

Appendix A Kappa statistic calculation 


\begin{tabular}{|c|c|c|c|c|c|c|c|c|}
\hline & 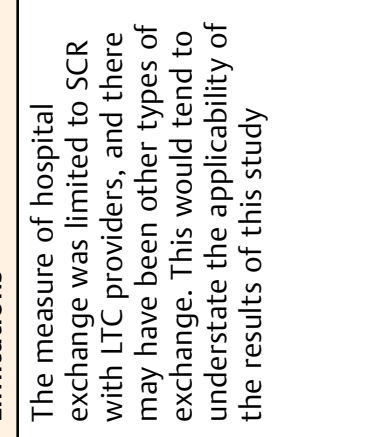 & \multicolumn{3}{|l|}{ 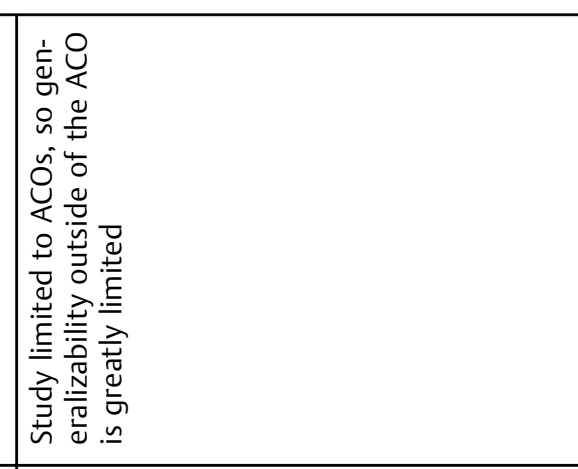 } & \multicolumn{4}{|c|}{ 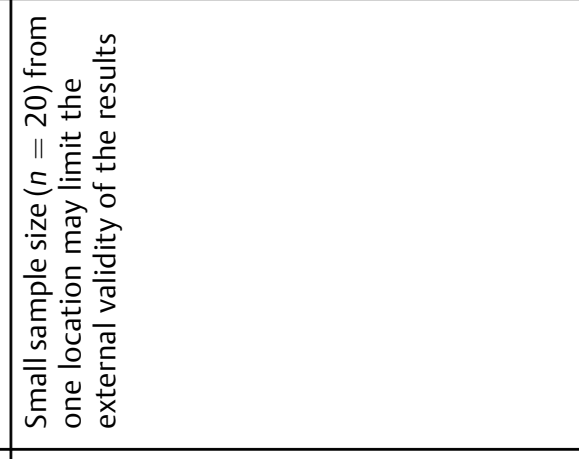 } \\
\hline & 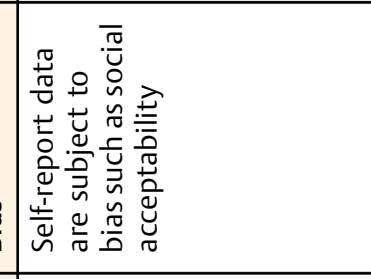 & \multicolumn{3}{|l|}{ 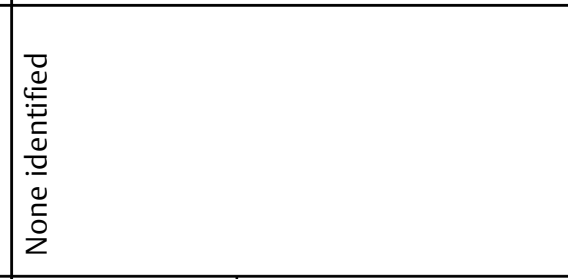 } & \multicolumn{4}{|c|}{ 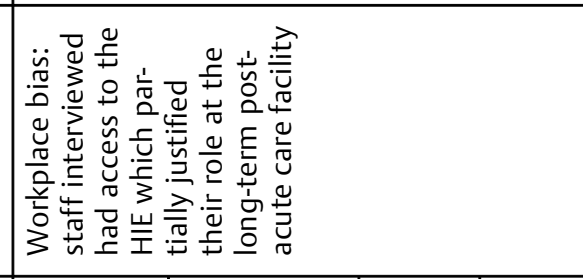 } \\
\hline & 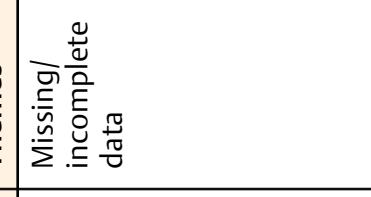 & 苍 & \multicolumn{2}{|l|}{ 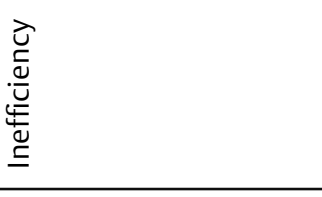 } & 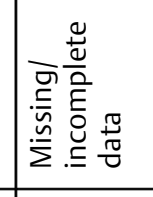 & 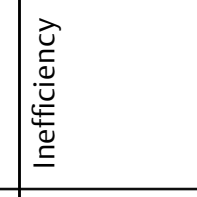 & 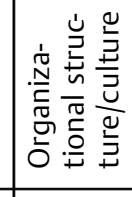 & \\
\hline & 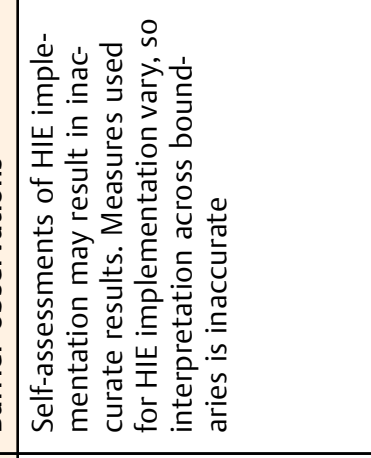 & 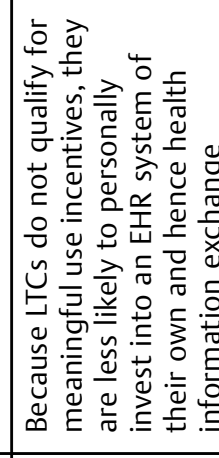 & \multicolumn{2}{|c|}{ 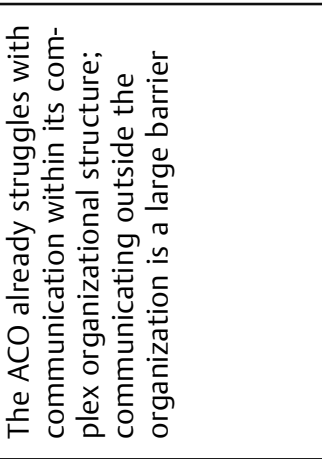 } & 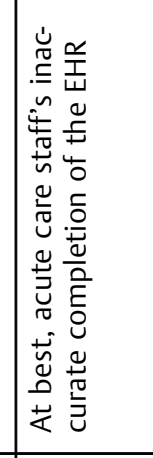 & 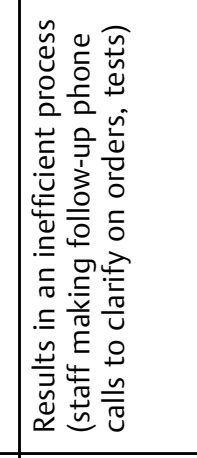 & 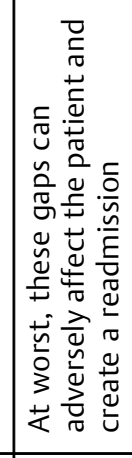 & \\
\hline & 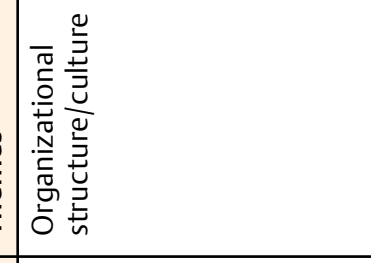 & 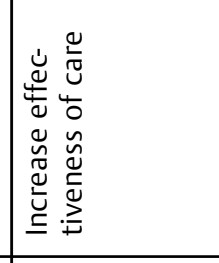 & & 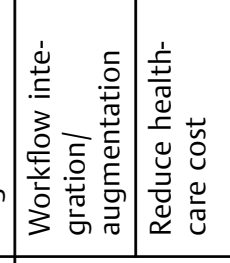 & 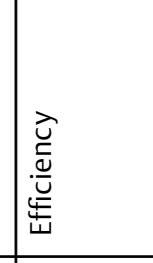 & 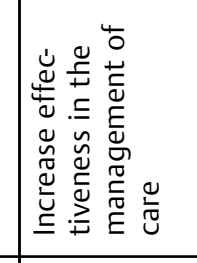 & 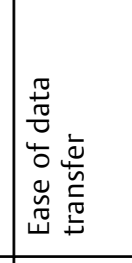 & 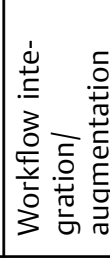 \\
\hline & 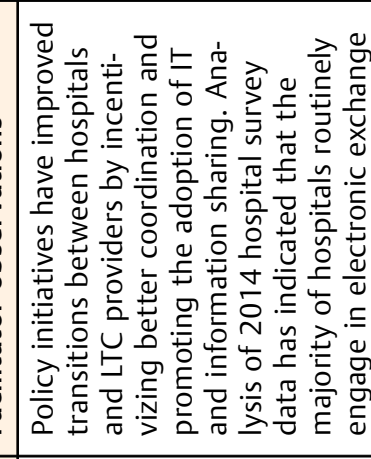 & 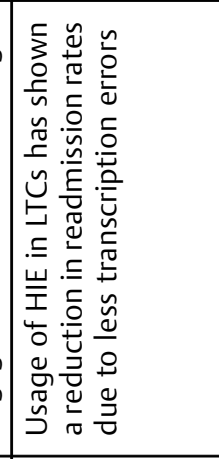 & 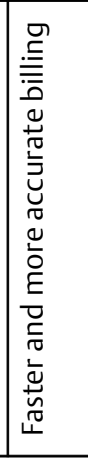 & 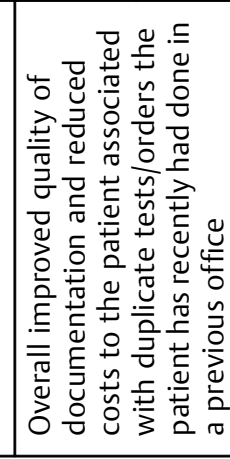 & 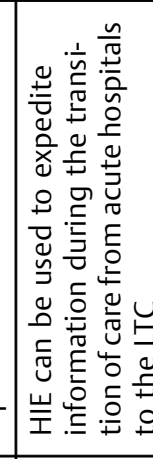 & 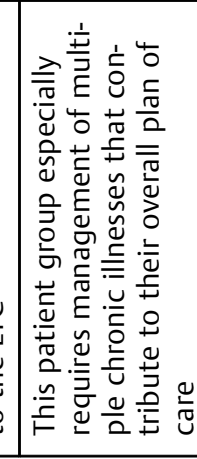 & 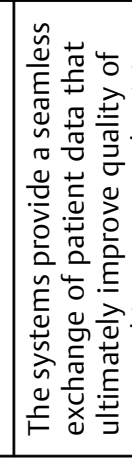 & 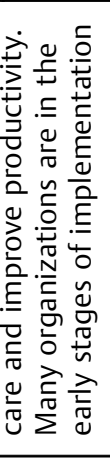 \\
\hline & 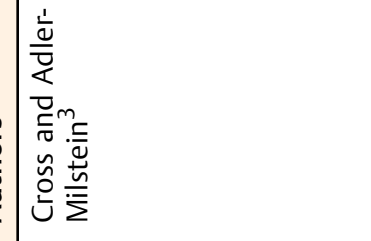 & \multicolumn{3}{|l|}{ 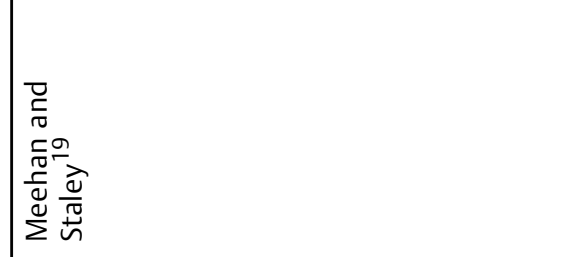 } & \multicolumn{4}{|l|}{ 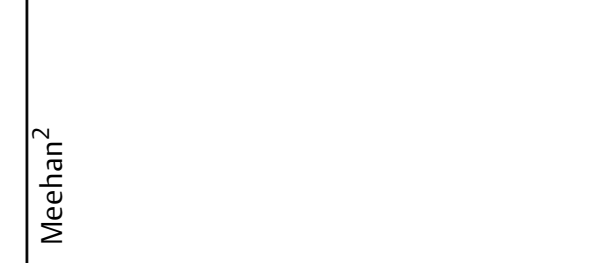 } \\
\hline
\end{tabular}




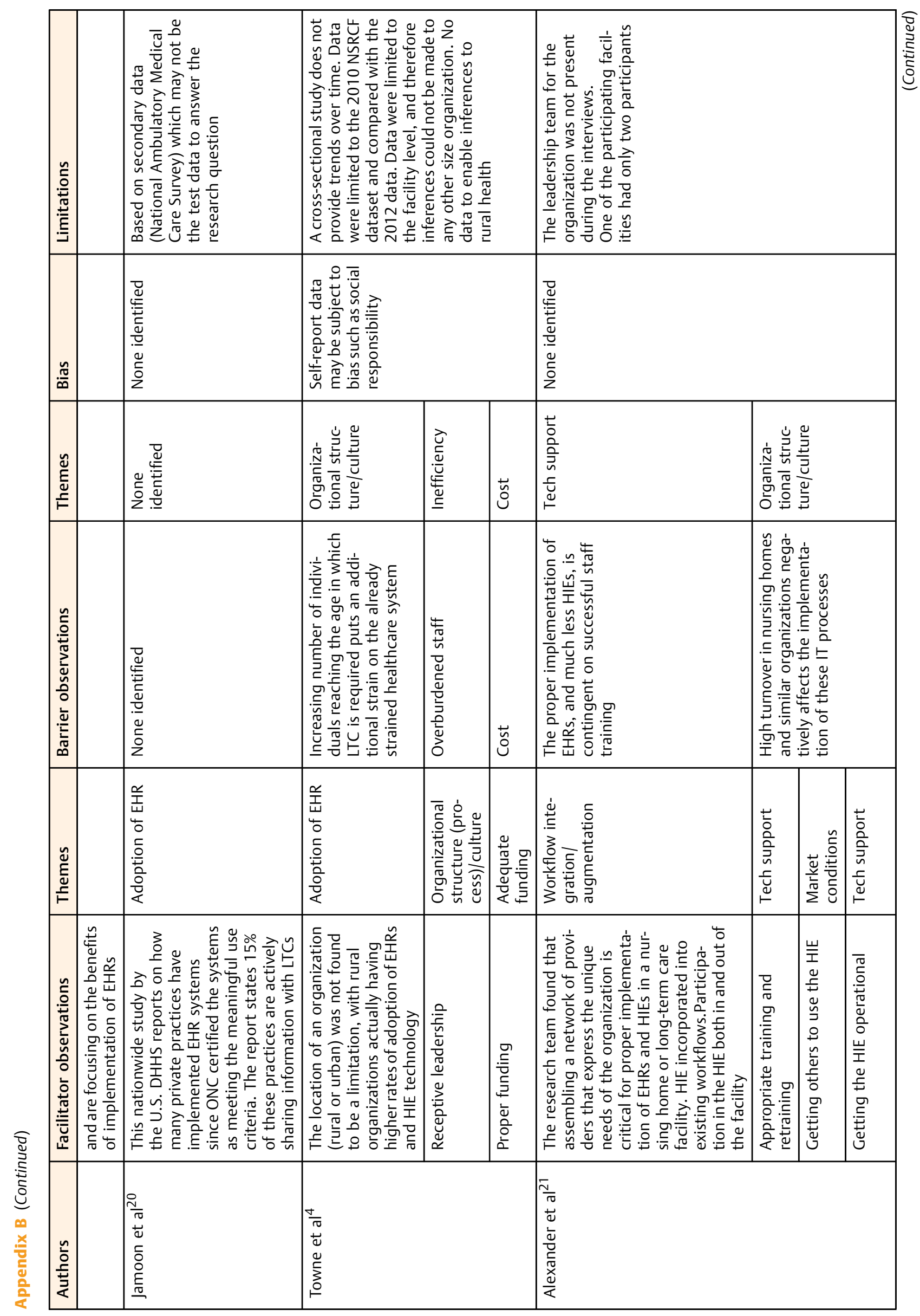




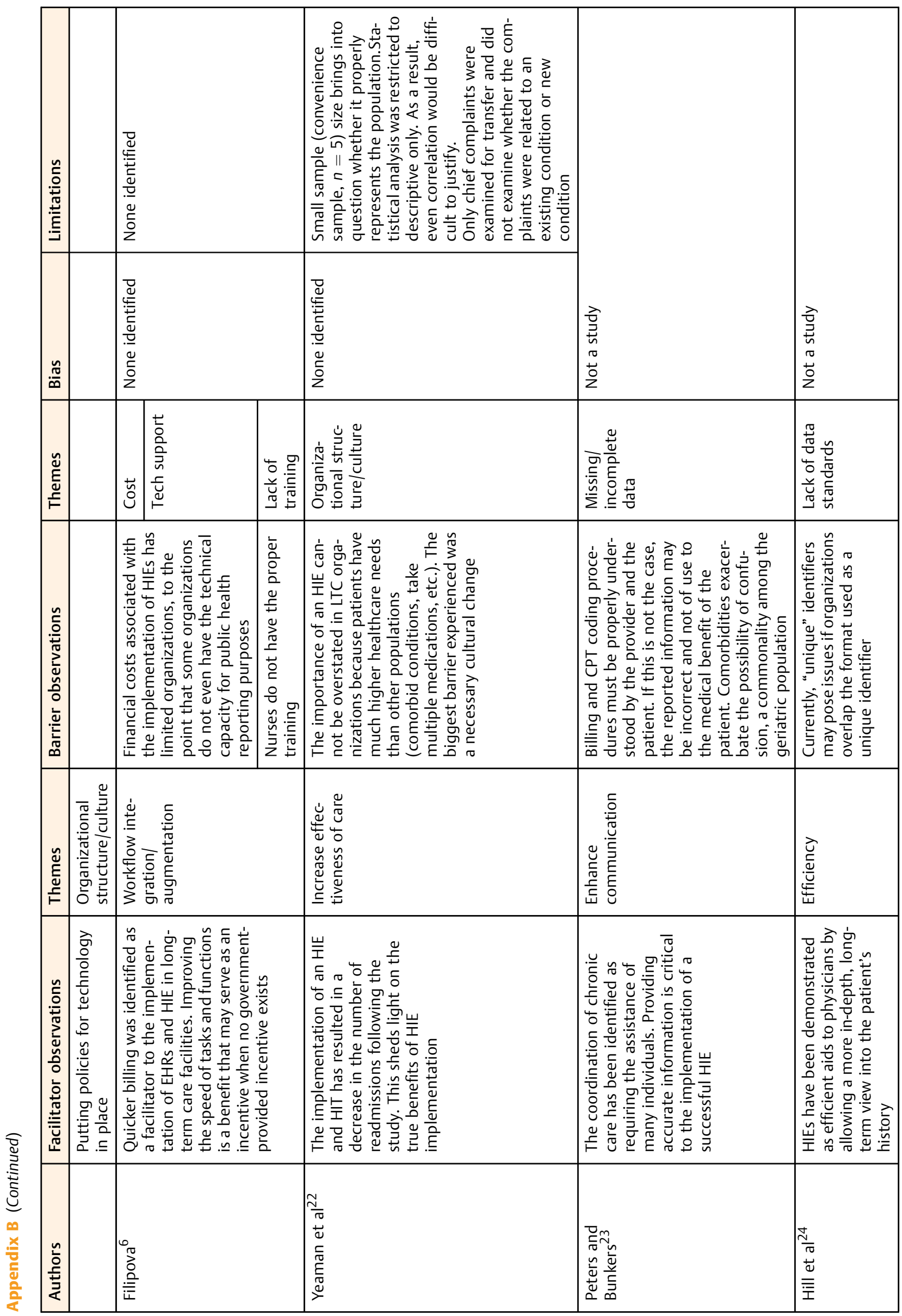




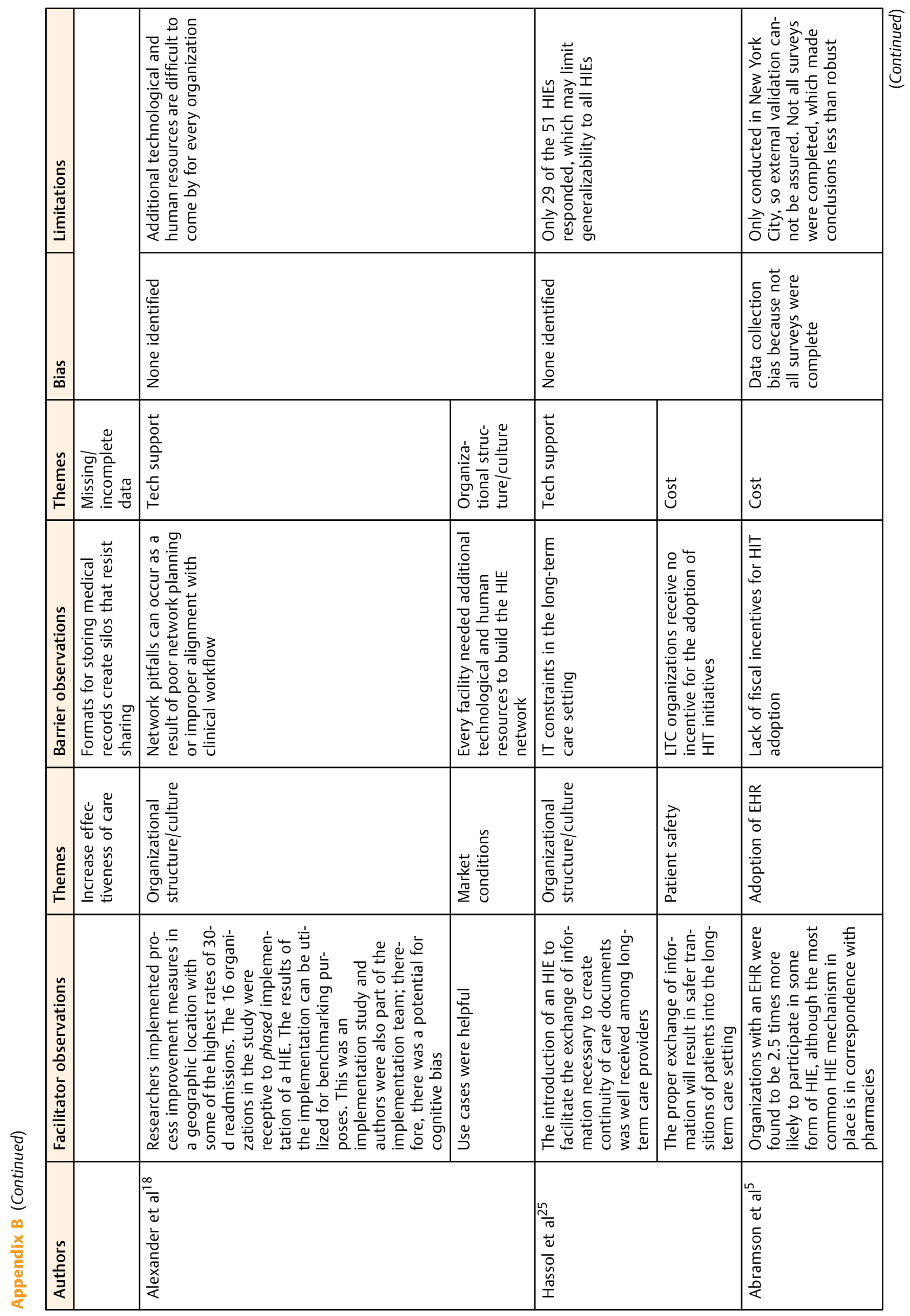




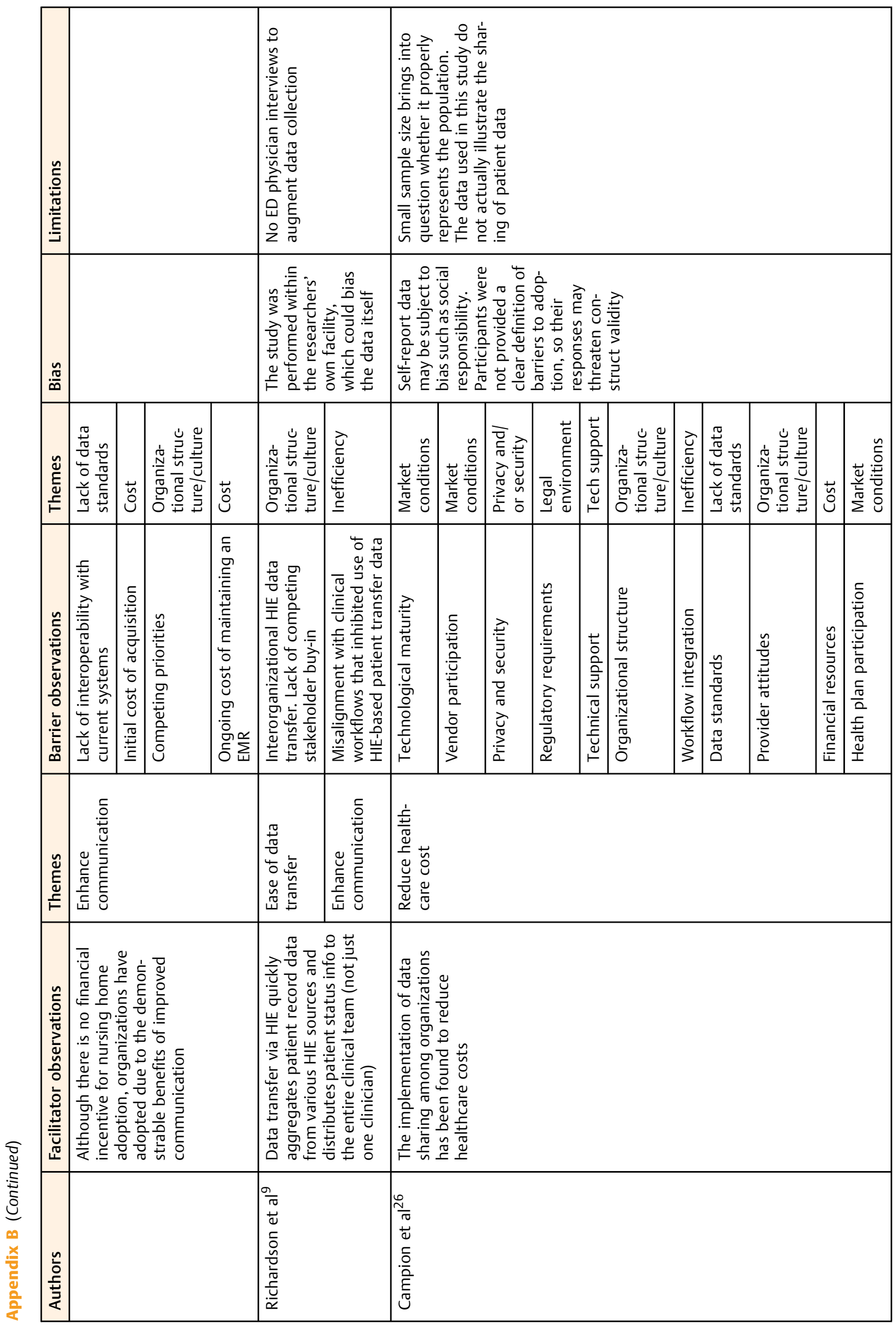




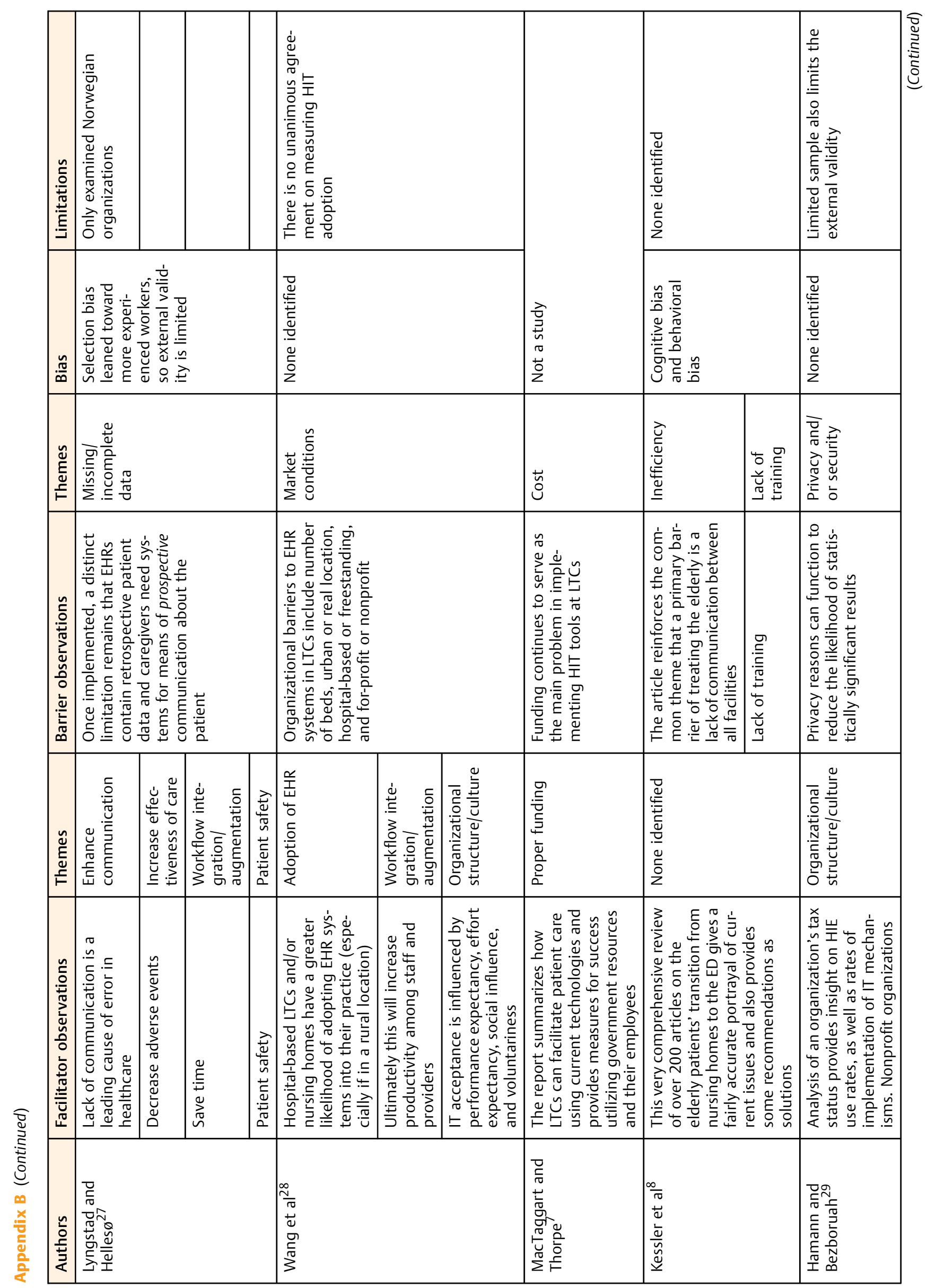




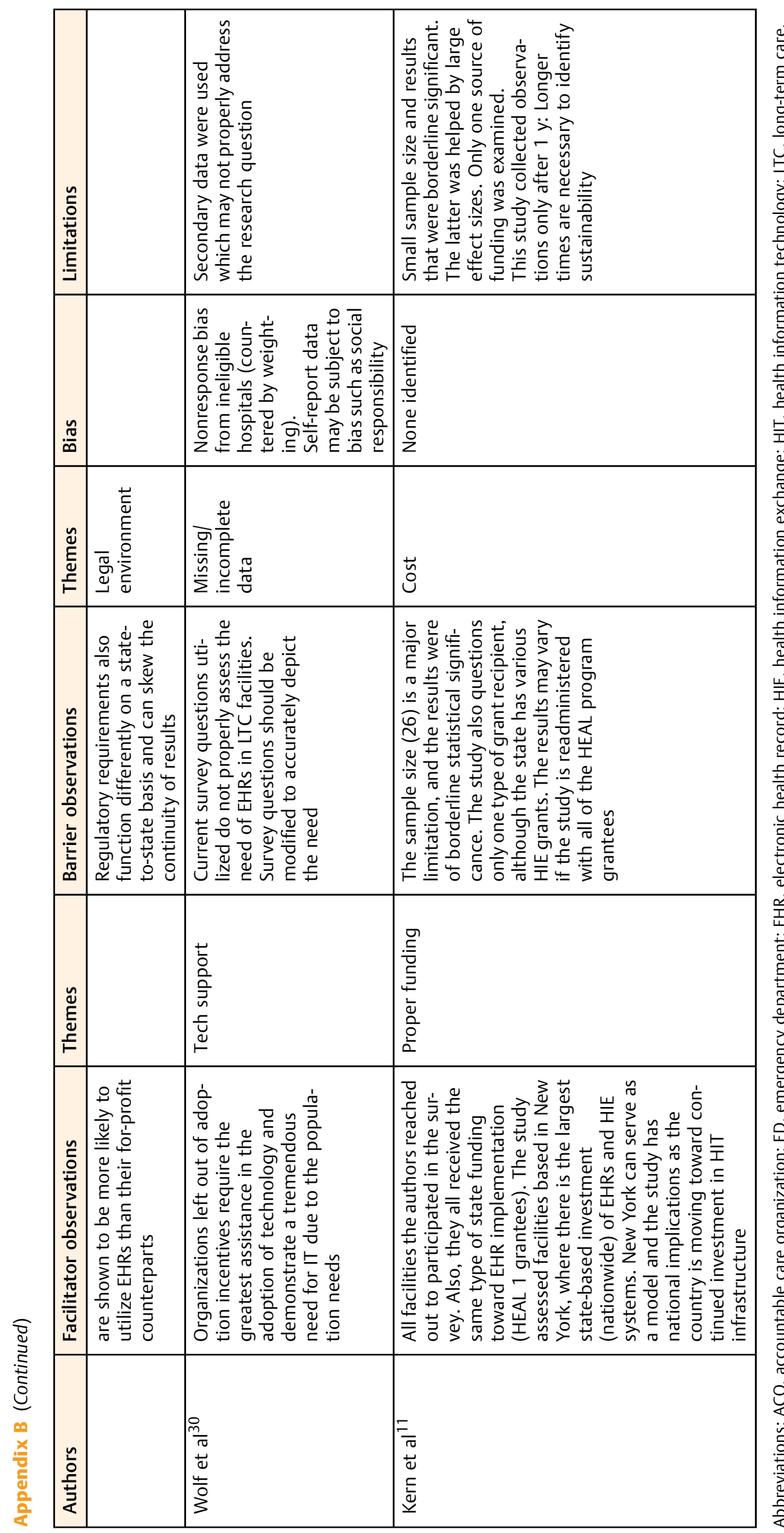

Applied Clinical Informatics Vol. 9 No. 4/2018 


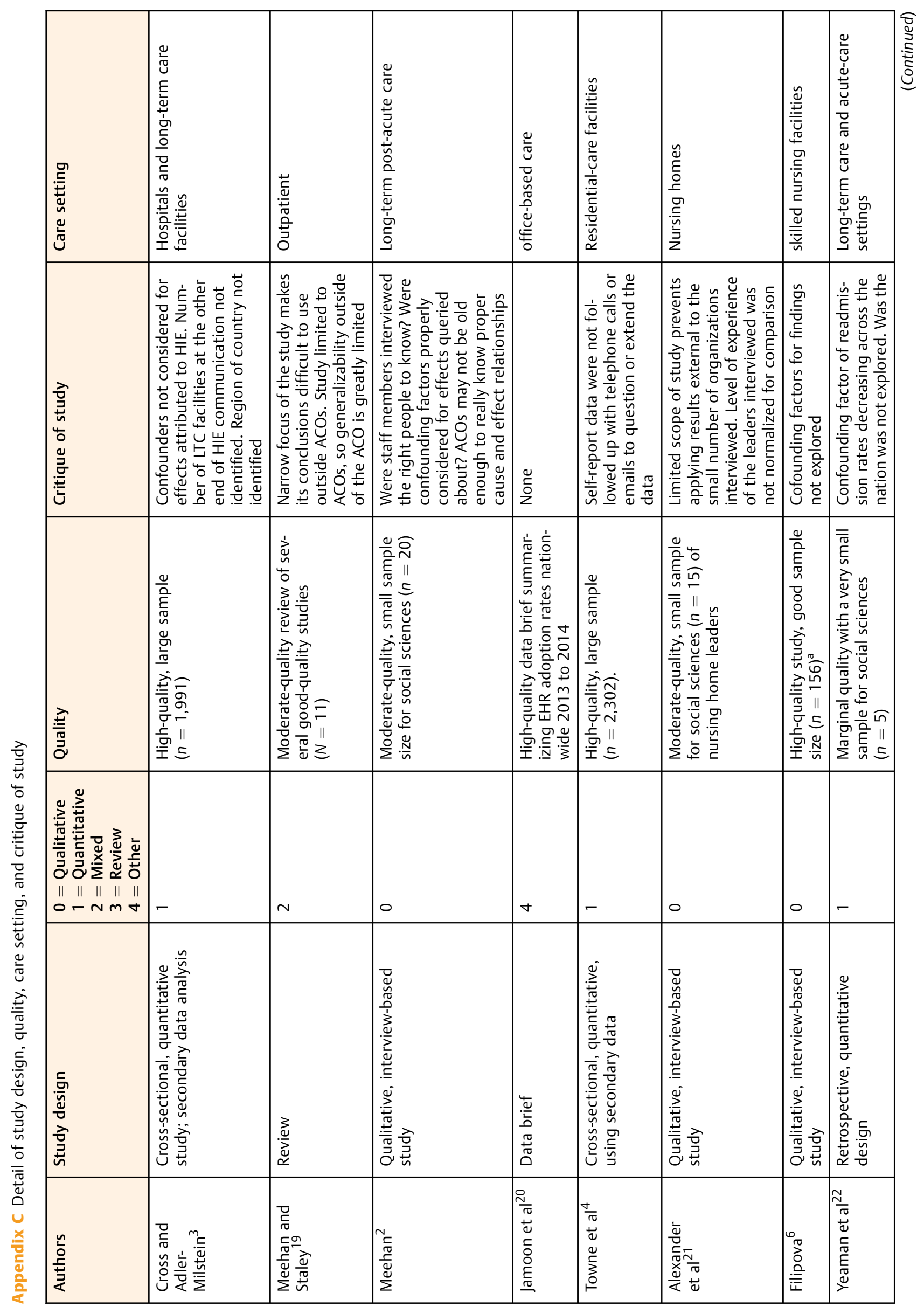




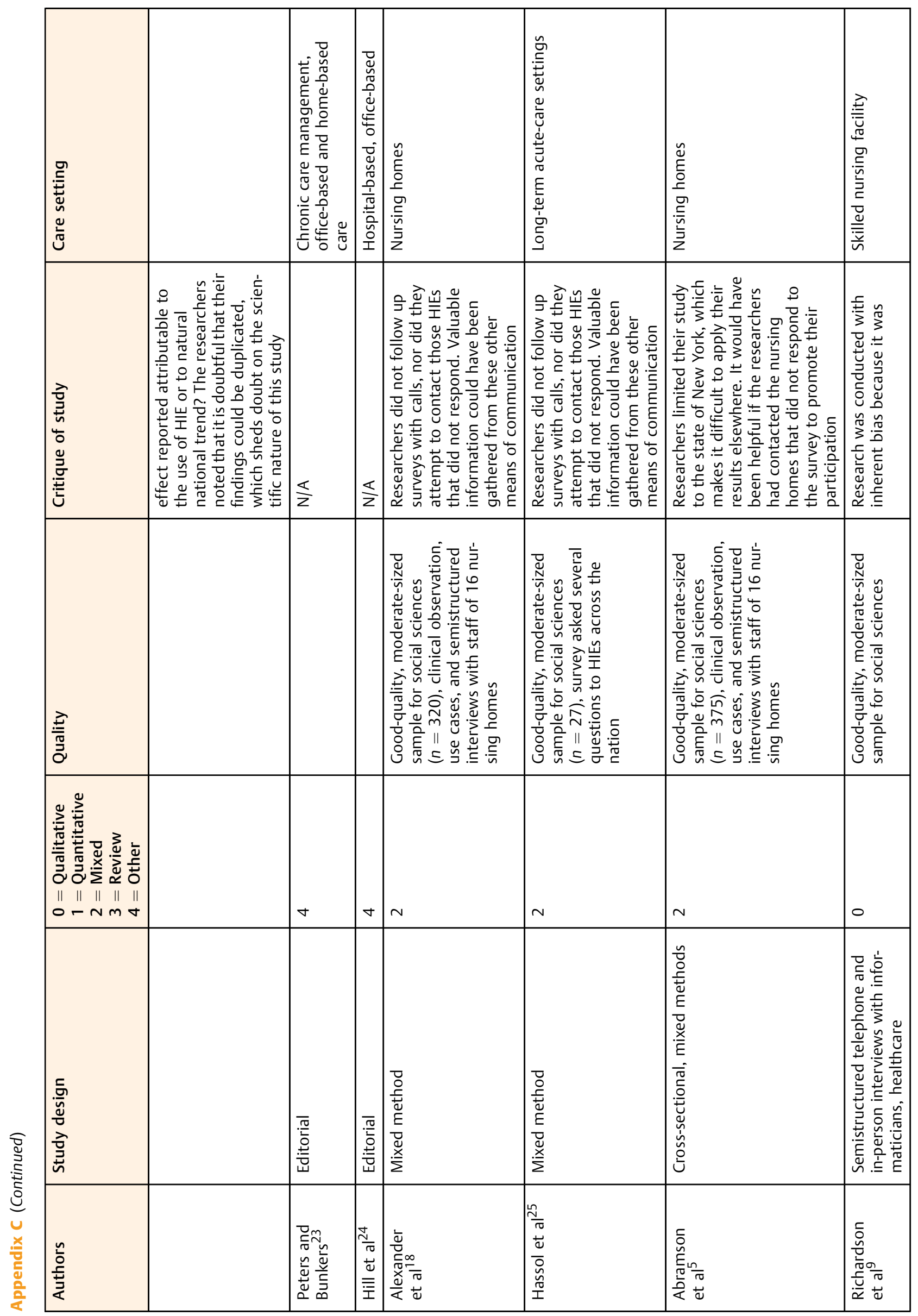




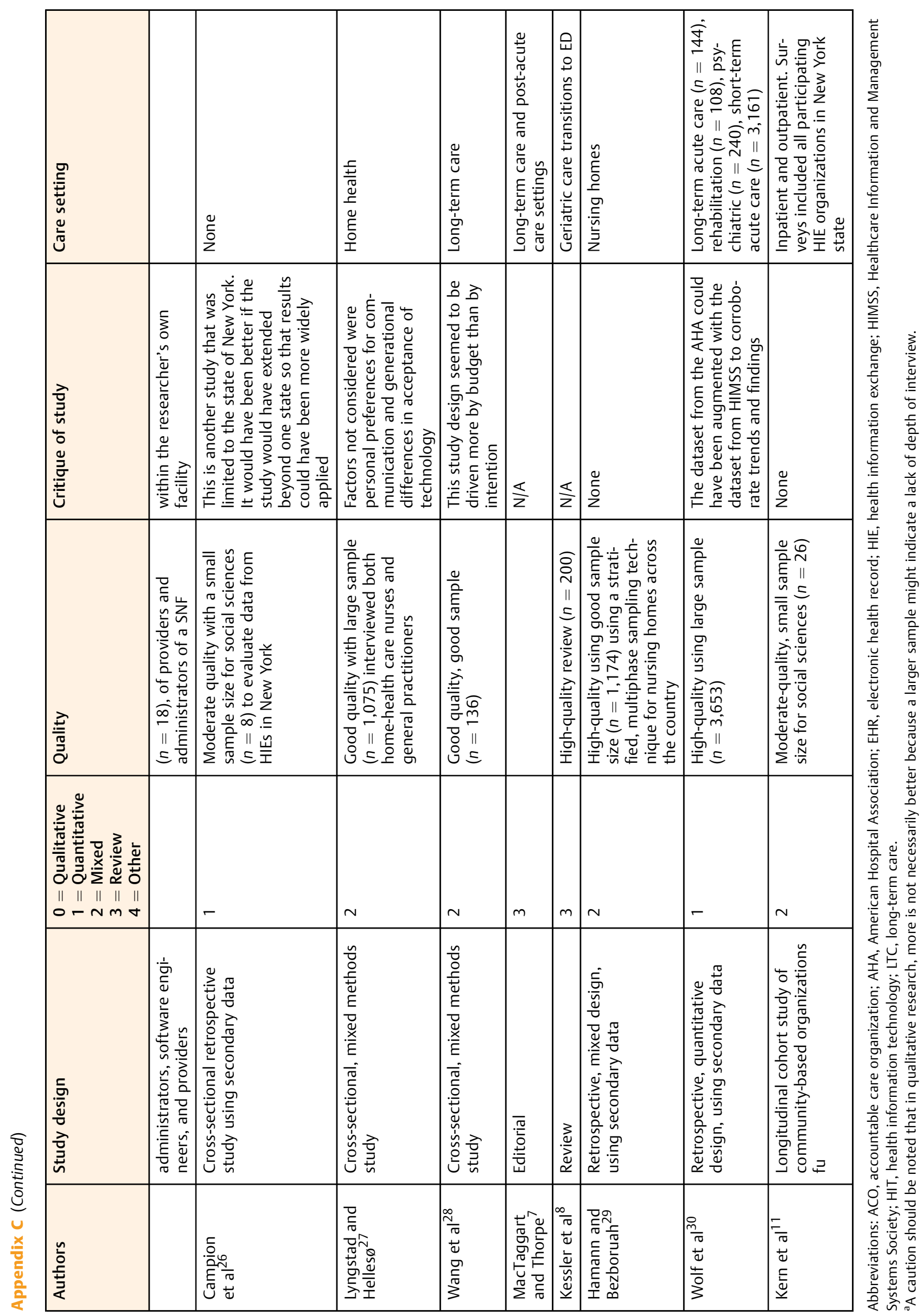

